\section{The development of visually guided reaching in monkeys reared without sight of the hands*}

\author{
RICHARD D. WALK and ELIZABETH K. BOND \\ George Washington University, Washington, D.C. 20006
}

In a modified replication of a study by Held and Bauer, two monkeys were reared without sight of the hands. The animals were taught to reach toward a dowel stick they could see. When the hands were uncovered, neither prolonged hand watching nor inability to reach toward objects was observed. The animals reached toward gross objects on the first day and gradually improved. Thus, visual deprivation of the sight of the hands would not appear to hinder visually guided reaching as much as the Held-Bauer study would indicate.

This study is a modified replication of an experiment by Held \& Bauer (1967). Their study used an ingenious procedure to rear infant monkeys without sight of the hands for 34 days. The hypothesis was that visually guided behavior would be severely affected because the hands could not be seen during early development. When the monkeys' hands were uncovered, Held and Bauer observed prolonged hand watching that interfered with reaching toward objects. Only after extensive experience with viewing objects in relation to the moving hands did accurate visually guided reaching develop.

Prior to the uncovering of the hands, their monkeys had been taught to extend an arm when a feeding bottle was presented. We felt that their procedure essentially taught the animals that visual objects had no relation to the position of the hands, since their animals were not required to reach in the direction of the bottle. Consequently, we modified their procedure to teach our animals to reach out toward and grasp a dowel stick that could be both seen and felt. The animal could not, however, see his hand touch the dowel. Both experiments, then, deprived the animal of the normal conjunction of vision and touch, but theirs taught the animal to reach out anywhere in space to the sight of the object, while ours taught a response related to the sight of the object. If visual-motor feedback of the hand in relation to the object is necessary for the development of visually guided reaching, our results should be no different from theirs.

* Presented at the Eastern Psychological Association meetings, Washington, D.C.. April 19, 1968. The study was supported, in part, by a grant from the National Institutes of Health (1-T01-HD-00203) and the National Seience Foundation (GY-2765). The assistance of Toni Falbo and Caroline Lebowitz in this experiment is gratefully acknowledged.
Our first purpose, then, was to determine if experience in which vision and prehension were correlated, but the hand was not visible, would be sufficient for the development of visually guided reaching.

A second purpose, developed during the experiment, was to study the sequence of the development of complex visually guided reaching behavior. A number of tasks were devised to assess accurate grasping and retrieval of objects, tasks where progress could be assessed, we hoped, in an accurate and objective manner. SUBJECTS

Two male macaque monkeys, one a cynamolgus macaque, the other a stumptailed (speciosa) macaque, served as Ss.

\section{PROCEDURE}

Our procedure was almost identical to that of Held and Bauer. Each week-old ${ }^{1}$ monkey was taken from the mother and placed in a rearing apparatus where it could feed itself ad lib but could not see its hands. From approximately 16 to 35 days of age, each animal received a daily conditioning session. A dowel stick was held vertically in front of the apparatus so that it extended upward into the visual field and downward into the tactual field. The monkey was required to reach out and grasp the immobile dowel in order to receive a sip from the feeding bottle. The stick was held up to the left and right of the animal, as well as directly in front of him, and he reached out with one arm at a time, while the other arm was restrained. Thus, the animal was required to reach to a specific locus, and this point was coordinated with the point to which the animal directed its gaze. At 36 days of age, each monkey was allowed to view his right hand and arm for $1 \mathrm{~h}$ and was presented various objects. After 20 days of training, $1 \mathrm{~h}$ a day, with the right hand, the left hand was exposed alone for most of the hour-long testing period. At the end of this session, the monkey was allowed to view both hands simultaneously in order to assess hand dominance. This procedure of viewing the left hand and then both hands was continued for 10 days or until the animal had accomplished all of the visual reaching tasks set for him.

The visual reaching procedure was worked out during the course of experimentation until a standard procedure was evolved. Standard objects presented to the monkey were the following: the feeding bottle, an orange fuse on the end of a string, and a Lifesaver or a raisin presented either on the end of the dowel stick or alone. The Lifesaver and raisin functioned both as objects and as rewards. The dowel stick was presented to the animal in six orientations, and the animal on some trials grasped the dowel to obtain the reward by chewing the end of the stick; on other trials the animal plucked the object from the end of the dowel and transported it to his mouth. Both Lifesaver and raisin were also held up in the E's fingers or presented flat on the E's palm.

The sequence of performance observed with all of these visual stimulus objects was the following: accurate reaching for the objects, correct grasping of the objects, carrying of the objects to the mouth, and placing of the objects in the mouth.

\section{RESULTS}

Contrary to the findings of Held and Bauer, the initial reach with the monkey's right arm was not interrupted by hand watching. Also, the animals did not engage in extensive hand watching during any of the testing sessions. We estimate that the animals spent not more than $4 \%-5 \%$ of the time looking at their hands alone (as distinct from the obvious fact that they had to see their hands when reaching for an object). The same lack of hand watching was noted when the left arm and hand were exposed.

Although the initial visual reaches were awkward, the animals were able to reach for fairly large objects, such as the feeding bottle and the dowel stick, on the first day of testing with the right hand. Performance with the left hand was also rather awkward at first, but it was much superior to initial performance with the first hand tested. That is, while perfect transfer of training from right to left hand could not be claimed, considerable savings were observed.

When both hands were tested simultaneously, the hand first exposed was quite dominant for a few days, but the animal soon developed equal facility with either hand. 
A hierarchy of difficulty was found among the tasks that the monkeys were required to perform. In general, the following tasks required increasing amounts of practice for successful performance: (1) Gross reaching-simple reaching and grasping of objects such as the feeding bottle. Successful, though awkward, performance on this task was observed on the first day of testing. (2) Precise reaching and grasping-an example here is the dowel stick presented in six different orientations. This more refined task required more practice than did simple, gross reaching. (3) Taking objects off the dowel stick. The animal could hold onto the dowel and chew off the reward before he could pick it off with his hand. (4) Grasping small objects from the E's fingers or from the palm of his hand. The animal was able to grasp an object held up in space before he could take an object from the E's palm. (5) Getting small objects into the mouth with the animal's own hand. This was more difficult with the raisin than with the Lifesaver and was the last task to be performed successfully.

From the above, we concluded that locating an object in space with the hand was the easiest task for the monkey, grasping and transporting it successfully was more difficult, and most difficult of all was handling and getting to the mouth an object, such as the raisin, which was hidden momentarily from sight by the animal's hand.

DISCUSSION AND CONCLUSIONS

We will discuss three points that derive from our study. The first point compares our results with Held and Bauer's and discusses the discrepancies, the second refers to experimental controls, and last, we have a tentative formulation of three developmental subsystems of visually guided reaching behavior based on the tasks we devised to study it.

(1) Held and Bauer's Results As Compared to Ours

The exact reason why our results differ from theirs is unknown. The conclusions derived from our results are preliminary, based as they are on only two animals and on our first exploration of visually guided behavior in the infant monkey. We may, however, tentatively conclude: first, that being deprived of viewing the hands does not hinder the development of visually guided reaching as much as one would conclude from the Held-Bauer study; second, that our experiment seems to demonstrate that, contrary to Held and Bauer, extensive hand watching is not necessary for the development of visually guided reaching; and third, that some experience in the immediate coordination of visual and tactual activities, as was provided in our conditioning procedure, may be sufficient to develop gross visually guided reaching behavior. The sight of the moving hand related to the object may not be necessary.

All of this means that the Held-Bauer procedure (and the "kitten carousel" one by Held-Hein, 1963) may have degraded coordinated visual behavior, while ours, though it also prevented tactual-visual contact, may have preserved it.

\section{(2) Controls}

Both studies are inadequately controlled. The only difference between experimental and control groups should be that difference produced by the experimental variable: sight of the hands during rearing. In this experiment, the proper control animal would be reared in an apparatus similar to the Held-Bauer one that restricts locomotion and requires that the animal feed itself. This control apparatus might have a transparent rather than an opaque collar-barrier-the animal could see its hands but would be in every other respect similar to the animals of the present study. Another important control procedure would restrict locomotion but would allow sight and free use of the hands. The apparatus used could have a thin collar but no board-barrier between the animal's hands and its head. A last control would, of course, consist of careful observation of a monkey reared away from its mother but in a cage that would allow for adequate locomotion. All of these controls, when taken together, would give some notion of the extent to which each of the variables-restriction of locomotion, restriction of contact between hands and head, and restriction of visual input from the sight of the moving hands-would influence the development of normal visually guided behavior. This would increase our understanding of the development of visual reaching and make it possible to relate this information to that obtained from other deprivation studies and to a general theory of development.

(3) Subsystems of Visually Guided Behavior

As a result of our work with a variety of tasks during the course of the development of visually coordinated reaching, we wish to posit three related but somewhat independent subsystems.

(a) Perceptual-motor subsystem. This sytem enables the animal to fixate on an object visually and to localize the object in space with the hand. This seems to be the most primitive of all of the subsystems, although some portions of it may develop more slowly than others. For example, an object on a flat surface parallel to the floor may be more difficult to localize, and the ability to locate it may develop later perceptually, than an object held up in space.

(b) A motor-retrieval subsystem. This includes the ability to grasp objects correctly, with the thumb in opposition to the other fingers, and to make refined manipulations with the hands and arms (cf. Hines, 1942). It also includes the capacity to convey a visible object through space to some goal, such as the mouth.

(c) A cognitive subsystem. This system includes the capacity to work with or manipulate objects momentarily lost from sight. A raisin in the monkey's hand, for example, is likely to be "forgotten" and dropped to the floor before it is conveyed to the mouth.

In conclusion, our procedure has secured results different from Held and Bauer's, a lack of hand watching and less disturbance of visually coordinated behavior. However, we feel that our most important contribution may be a demonstration of the need for a more refined analysis of the complexities of visually coordinated behavior.

\section{REFERENCES}

HELD, R., \& BAUER, J. A., JR. Visually guided reaching in infant monkeys after restricted rearing. Science, 1967, 155, 718-720.

HELD, R., \& HEIN, H. Movement-produced stimulation in the development of visually guided behavior. Joumal of Comparative \& Physiological Psychology, $1963,56,872-876$.

HINES, M. The development and regression of reflexes, postures, and progression in the young macaque. Contributions to Embryology, 1942,30,153-209 (Whole No. 196)

\section{NOTE}

1. The animals were left with the mother for 1 week on the advice of Dr. Joseph Bauer. 